\title{
Atypical antipsychotic drugs induce derangements in glucose homeostasis by acutely increasing glucagon secretion and hepatic glucose output in the rat
}

\author{
G. C. Smith • C. Chaussade • M. Vickers • J. Jensen • \\ P. R. Shepherd
}

Received: 20 July 2008 / Accepted: 13 August 2008 / Published online: 9 October 2008

(C) Springer-Verlag 2008

\begin{abstract}
Aims/hypothesis Use of the second-generation antipsychotic drugs (SGAs) results in the development of obesity and a type 2 diabetes-like syndrome. We hypothesised that, in addition to the insulin resistance associated with the obesity, the SGAs might have acute effects on glucose metabolism that could contribute to the derangements in glucose metabolism.

Methods We investigated the effects of therapeutically relevant levels of three different antipsychotic medications (haloperidol, quetiapine and clozapine) on glucose tolerance, measures of insulin resistance and hepatic glucose production, and on insulin and glucagon secretion in rats.
\end{abstract}

Electronic supplementary material The online version of this article (doi:10.1007/s00125-008-1152-3) contains supplementary material, which is available to authorised users.

G. C. Smith $\cdot$ C. Chaussade $\cdot$ P. R. Shepherd $(\triangle)$

Department of Molecular Medicine and Pathology and Maurice Wilkins Centre for Molecular Biodiscovery, University of Auckland,

Private Bag 92019,

Auckland, New Zealand

e-mail: peter.shepherd@auckland.ac.nz

\section{Vickers}

Liggins Institute and National Research

Centre for Growth and Development,

University of Auckland,

Auckland, New Zealand

J. Jensen

Department of Physiology,

National Institute of Occupational Health,

Oslo, Norway
Results We found that these drugs induce impaired glucose tolerance in rats that is associated with increased insulin secretion (clozapine $>$ quetiapine $>$ haloperidol) but is independent of weight gain. However, Akt/protein kinase B activation is normal, and at these levels of drug there was no effect on insulin action in fat cells or soleus muscle, and no effect on insulin sensitivity as evaluated by insulin tolerance tests. We show that clozapine induces increased glucose levels following pyruvate and glycerol challenges, indicating an increase in hepatic glucose output (HGO). Increased HGO would in turn increase insulin release and would explain the apparent phenotype mimicking insulin resistance. We provide evidence that this effect could at least in part be mediated by a stimulation of glucagon secretion. Conclusions/interpretation Our findings indicate that SGAs can cause acute derangements in glucose metabolism that are not caused by a direct induction of insulin resistance but act via an increase in glucagon secretion and thus stimulation of hepatic glucose production.

Keywords Clozapine · Glucagon · Gluconeogenesis · Hepatic glucose production · Schizophrenia .

Type 2 diabetes

$\begin{array}{ll}\text { Abbreviations } \\ \text { DEXA } & \text { dual-energy x-ray absorptiometry } \\ \text { GlycTT } & \text { glycerol tolerance testing } \\ \text { GTT } & \text { glucose tolerance testing } \\ \text { HGO } & \text { hepatic glucose output } \\ \text { ITT } & \text { insulin tolerance testing } \\ \text { PKB } & \text { pyruvate kinase B } \\ \text { PTT } & \text { pyruvate tolerance testing } \\ \text { SGA } & \text { second-generation antipsychotic }\end{array}$




\section{Introduction}

Antipsychotic medications are widely used in the treatment of schizophrenia and other forms of psychosis. The drugs fall into two main groups, known as typical or atypical antipsychotics. The most widely used typical medication is haloperidol, which has high antagonist affinity for type 2 dopamine (D2)-like receptors (D2, D3 and D4 receptors) but causes extrapyramidal side effects [1]. The atypical or second-generation antipsychotic medications (SGAs), such as quetiapine, olanzapine and clozapine are thought to be more targeted, therapeutically, because of their low incidence of extrapyramidal side effects resulting from their low affinity for D2 receptors. However, a major side effect of SGAs is the development of symptoms similar to type 2 diabetes [1-5]. It is widely assumed that this is caused by peripheral insulin resistance following excessive weight gain observed with people prescribed these drugs [6-8].

Despite the profound effects of SGAs on glucose metabolism, surprisingly little is known about the direct effects of these on glucose homeostasis. A study showed a slight increase in blood glucose level after clozapine treatment but the glucose measurements were obtained from anaesthetised rats, limiting the interpretation of the results [9]. Similarly, another group investigated the effect of clozapine on glucose tolerance, but used trunk blood following decapitation, which in itself is normally associated with elevated blood glucose concentrations [10]. Researchers have also reported that olanzapine caused insulin resistance in L6 skeletal muscle cells, but this effect is only apparent using a 100 -fold $(100 \mu \mathrm{mol} / \mathrm{l})$ higher concentration of the drug than we would see in the clinical setting [11]. Finally, another study observed no effect on glucose tolerance with olanzapine; however, the drug was administered in food and the rats were subsequently fasted overnight before performing the test [1]. Because antipsychotic drugs have a very short half-life in the rodent $(<2 \mathrm{~h})$ the glucose challenge was performed on rats with no or very low concentration of the drug [12]. Thus, it has become increasingly unclear whether the association between schizophrenia and type 2 diabetes is of aetiological significance to schizophrenia or an off-target effect of antipsychotic medication.

The current study was undertaken to determine whether therapeutically relevant concentrations of SGAs are having acute effects on the mechanisms regulating glucose metabolism. These studies show that while the SGAs induce rapid impairment of glucose metabolism, they do not acutely induce insulin resistance. Instead they acutely induce glucagon secretion and hepatic glucose output (HGO), which together will contribute to the increase in glucose levels induced by SGAs. We propose that these acute effects of SGAs contribute to the diabetes-like phenotype observed in humans.

\section{Methods}

Animal model Male Sprague-Dawley rats (VJU, Auckland, New Zealand) were weight-matched into the following groups: vehicle control (5\% [vol./vol.] acetic acid, $\mathrm{pH} 6)$, haloperidol $(0.25 \mathrm{mg} / \mathrm{kg}$; Sigma, Castle Hill, NSW, Australia), quetiapine (10 mg/kg; AstraZeneca, Auckland, New Zealand) or clozapine $(10 \mathrm{mg} / \mathrm{kg}$; Douglas Pharmaceuticals, Auckland, New Zealand). Each group received a daily s.c. injection of the vehicle control or antipsychotic drug for either 1-2 $\mathrm{h}$ (acute), 7 days (sub-chronic) or 28 days (chronic). All rats had free access to a standard chow diet (Global Rodent Diet; Teklad, Madison, WI, USA; 18\% protein, $5 \%$ fat, digestible energy $3.4 \mathrm{~kJ} / \mathrm{g}$ ) and water. Rats used for tissue collection and plasma drug quantification were fasted overnight and $2 \mathrm{~h}$ after their drug injection were anaesthetised with sodium pentobarbitone $(60 \mathrm{mg} / \mathrm{kg})$ and killed. All work was approved by the Animal Ethics Committee of the University of Auckland.

Measurements Body fat composition was assessed using dual-energy X-ray absorptiometry (DEXA) (Lunar Hologic; GE Medical Systems, Belgium). Food intake and body weight were measured daily. Blood glucose levels were determined using an Accu-Chek Performa blood glucose meter (Roche, Auckland, New Zealand). Plasma insulin and glucagon levels were measured by the Mercodia Ultrasensitive rat insulin ELISA (Mercodia, Uppsala, Sweden) and Linco glucagon RIA (Linco, Billerica, MA, USA), respectively.

Plasma drug quantification Drug quantification was determined using HPLC (Alliance 2475; Waters, Milford, MA, USA) $[13,14]$. In brief, $4 \mu 1$ of internal standard $(100 \mu \mathrm{g} / \mathrm{ml}$ amoxapine) was added to $250 \mu 1$ of rat plasma. Fifty microlitres of sodium hydroxide $(2 \mathrm{~mol} / \mathrm{l})$ was added and mixed thoroughly; $1.5 \mathrm{ml}$ hexane-isoamyl alcohol (98:2, vol./vol.) was added and mixed shaking for $15 \mathrm{~min}$ at room temperature. After centrifugation, the organic layer was removed and samples were back extracted in phosphoric acid. The hexane-isoamyl alcohol layer was removed using Hetovac (9 Pa, $-130^{\circ} \mathrm{C}, 2000 \mathrm{~g}$; Heto, Allerod, Denmark) for $20 \mathrm{~min}$. After centrifugation, $50 \mu \mathrm{l}$ of aqueous phase was added into the HPLC unit for a $20 \mu 1$ injection. An isocratic method was used with a flow rate of $0.7 \mathrm{ml} / \mathrm{min}$ using 95\% mobile phase (acetonitrile-water-tetramethylethylenediamine, 37.5/62.1/0.4 [vol./vol./vol.], pH 6.5 with $95 \%$ [vol./vol.] acetic acid) and 5\% [vol./vol.] acetonitrile and samples analysed at $254 \mathrm{~nm}$. Data analysis was assessed by Waters Millennium software, with the plasma 
drug quantification being determined from the calibration curve and internal standard.

Glucose tolerance testing (GTT) Rats were fasted for $16 \mathrm{~h}$. A baseline venous blood sample was collected from the tail tip. Rats were injected s.c. with the vehicle control or antipsychotic drug and after $1 \mathrm{~h}$ another blood sample and glucose measurement was taken (time 0). A bolus injection of glucose (2 g/kg of D-glucose; Merck, Auckland, New Zealand) was then injected i.p. At indicated times, blood was collected for glucose and plasma insulin quantification.

Insulin tolerance testing (ITT) Rats were fasted for $2 \mathrm{~h}$ and a basal blood glucose measurement taken. Rats were injected s.c. with the vehicle control or antipsychotic drug and after $1 \mathrm{~h}$ another blood glucose measurement was taken (time 0). A bolus injection of insulin $(0.75 \mathrm{U} / \mathrm{kg}$; Sigma) was then administered s.c. and blood glucose concentrations were taken at indicated time points.

Pyruvate tolerance testing (PTT) and glycerol tolerance testing (GlycTT) These used the same protocol as the GTT with the following exceptions. Following $1 \mathrm{~h}$ of antipsychotic drug injection, nine rats were injected with either pyruvate ( $2 \mathrm{~g} / \mathrm{kg}$; Sigma) or glycerol ( $2 \mathrm{~g} / \mathrm{kg}$; Sigma) with a further nine rats receiving no pyruvate or glycerol. For the clozapine/pyruvate or glycerol/octreotide experiments, rats were fasted for $16 \mathrm{~h}$ and injected with vehicle or clozapine. After $55 \mathrm{~min}$ of clozapine exposure half of the vehicle- and clozapine-injected groups received an s.c. injection of octreotide $(250 \mu \mathrm{g} / \mathrm{kg}$; Sandostatin, Novartis, Auckland, New Zealand). At $60 \mathrm{~min}$ after drug injection all rats received an i.p. injection of pyruvate $(2 \mathrm{~g} / \mathrm{kg})$ or glycerol $(2 \mathrm{~g} / \mathrm{kg})$ and a blood glucose concentration was taken after $90 \mathrm{~min}$ for pyruvate and $60 \mathrm{~min}$ for glycerol.

Octreotide experiment Rats $(260 \pm 20$ g) were weightmatched into groups: vehicle, vehicle plus octreotide, clozapine, or clozapine plus octreotide. Rats injected with octreotide received an s.c. injection $(250 \mu \mathrm{g} / \mathrm{kg}) 5 \mathrm{~min}$ before the vehicle or clozapine injection. After $1 \mathrm{~h}$, blood was taken from the tail tip for blood glucose determination and plasma for insulin and glucagon quantification.

Differentiation of 3T3-L1 cells 3T3-L1 fibroblasts were obtained from the American Type Culture Collection (Manassas, VA, USA) and induced to differentiate as described previously $[15,16]$.

Muscle incubations and glucose uptake assay Rats of about $140 \mathrm{~g}$ were anaesthetised with pentobarbital (10 mg i.p.; $50 \mathrm{mg} / \mathrm{ml}$ ), and the soleus muscles were dissected out and mounted on holders at their resting length and incubated as previously described [17]. After approximately 45 min preincubation, muscles were incubated for $30 \mathrm{~min}$ (for wortmannin only $10 \mathrm{~min}$ ) with the concentrations of antipsychotics as indicated in the Figures. Muscles were then incubated for an additional $30 \mathrm{~min}$ with insulin (and antipsychotics) for analysis of glucose uptake. Glucose uptake was measured for $30 \mathrm{~min}$, as previously described [18].

Analysis of Akt/pyruvate kinase B (PKB) serine 473 phosphorylation by immunoblotting After the indicated animal experiments, proteins were extracted in ice-cold lysis buffer (1\% [vol./vol.] NP-40; 10\% [vol./vol.] glycerol; $137 \mathrm{mmol} / 1 \mathrm{NaCl} ; 20 \mathrm{mmol} / 1$ TRIS-HCl, pH 7.4; $4 \mu \mathrm{g} / \mathrm{ml}$ aprotinin; $4 \mu \mathrm{g} / \mathrm{ml}$ leupeptin; $1 \mathrm{mmol} / 1$ 4-(2-aminoethyl) benzenesulfonyl fluoride; $4 \mu \mathrm{g} / \mathrm{ml}$ pepstatin; $10 \mathrm{mmol} / \mathrm{l}$ EDTA; $1 \mathrm{mmol} / 1$ EGTA, pH 8; 20 mmol/1 NaF; $1 \mathrm{mmol} / 1$ $\mathrm{Na}$ pyrophosphate and $1 \mathrm{mmol} / \mathrm{l}$ vanadate). The extracted proteins were quantified using a BCA Protein Assay Kit (Pierce, Auckland, New Zealand) and $50 \mu \mathrm{g}$ of protein was boiled for $7 \mathrm{~min}$ and separated by SDS-PAGE and transferred to polyvinylidene difluoride membranes. After blocking for $30 \mathrm{~min}$ at room temperature in $5 \%$ (wt/vol.) BSA/0.1\% (vol./vol.) Tween-20 in TRIS-buffered saline, membranes were incubated with polyclonal antibody specific for Akt/PKB phosphorylated on serine 473 (Cell Signaling Technologies, Auckland, New Zealand). The primary antibody was detected with a secondary antibody (horseradish peroxidase-conjugated goat anti-rabbit (Dako, Carpinteria, CA, USA) and enhanced chemiluminescence (ECL; Amersham Biosciences, Auckland, New Zealand). Detection was made using LAS 3000 (Fujifilm, Valhalla, NY, USA). The membranes were then stripped (2\% [wt./vol.] SDS; $100 \mathrm{mmol} / 1 \beta$-mercaptoethanol and $50 \mathrm{mmol} / 1 \mathrm{TRIS}$, $\mathrm{pH} 6.8$ ) for $30 \mathrm{~min}$ at $55^{\circ} \mathrm{C}$ and re-probed with a polyclonal total PKB antibody (Cell Signaling Technologies). Immunoreactivity was detected with ECL. All blots were quantified using multi-gauge V3.0 software (Fujifilm).

Statistical analysis Data are presented as means $\pm \mathrm{SEM}$ except where otherwise stated. Datasets with multiple time points and groups (e.g. GTT, PTT) were analysed by repeated-measures ANOVA with Fisher's post hoc test. $p<0.05$ was taken as significant.

\section{Results}

Blood levels of drugs Therapeutic concentrations in the circulation of patients is in the range of $5-10 \mathrm{nmol} / \mathrm{l}$ for haloperidol, 550-1,100 nmol/1 for quetiapine and 800 $1,050 \mathrm{nmol} / 1$ for clozapine [12, 19-21]. Similar blood levels in our rat model were achieved using $0.25 \mathrm{mg} / \mathrm{kg}$ 
haloperidol, $10 \mathrm{mg} / \mathrm{kg}$ quetiapine and $10 \mathrm{mg} / \mathrm{kg}$ clozapine (Electronic supplementary material [ESM] Table 1).

The effect of antipsychotic medication on body weight, body composition and food intake To determine whether antipsychotic medication induces excessive weight gain, we injected male Sprague-Dawley rats daily for 28 days with vehicle (5\% [vol./vol.] acetic acid, $\mathrm{pH} 6)$, haloperidol $(0.25 \mathrm{mg} / \mathrm{kg})$, quetiapine $(10 \mathrm{mg} / \mathrm{kg})$ or clozapine $(10 \mathrm{mg} /$ $\mathrm{kg}$ ). Rats injected with haloperidol or clozapine weighed significantly less than vehicle controls, whereas rats injected with quetiapine showed no difference in weight gain (ESM Fig. 1a). We also quantified the fat percentage of each group before and 28 days after the daily antipsychotic regime using DEXA. As shown in ESM Fig. $1 \mathrm{~b}$, the vehicle controls increased their fat percentage by $30 \%$ from the initial scan and quetiapine-treated by $40 \%$, with no change in fat percentage found with haloperidol or clozapine. The reduction in weight gain and fat percentage may be caused by either a decrease in food intake or an increase in energy expenditure. As shown

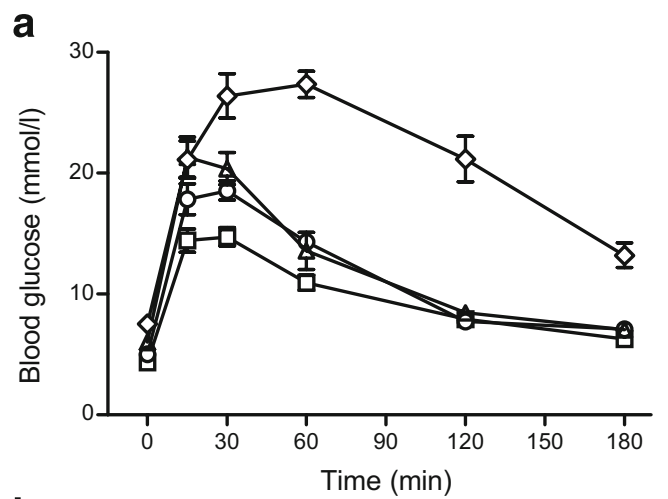

b

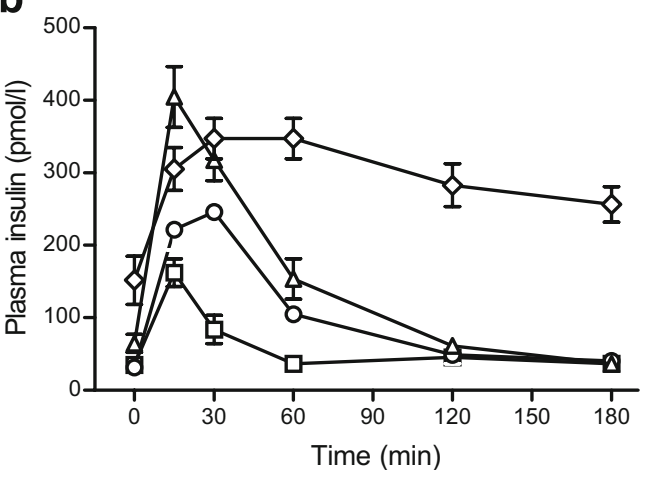

Fig. 1 The acute effect of antipsychotic drugs on glucose tolerance. Blood glucose levels (a) and plasma insulin levels (b) in a GTT performed $1 \mathrm{~h}$ after vehicle or drug injection (haloperidol, quetiapine or clozapine) (means $\pm \mathrm{SEM}, n=6$ per group). Squares, vehicle; circles, haloperidol $(p<0.01)$; triangles, quetiapine $(p<0.001)$; diamonds, clozapine $(p<0.001)$. Statistical significance determined by repeatedmeasures ANOVA, as compared with the vehicle control group in ESM Fig. 1c, rats injected with haloperidol or clozapine had a non-significant reduction in daily food intake, when compared with either the vehicle control or quetiapineinjected groups. Thus, any metabolic effect of antipsychotic medication will not be influenced by excessive weight gain or change in body composition, both of which are well known to cause insulin resistance and glucose metabolism alterations.

Acute effect of SGA drugs on GTT We first evaluated the effect of acute administration of SGAs in drug-naive animals on glucose and insulin levels during GTT. Both blood glucose and plasma insulin levels were significantly increased by $1 \mathrm{~h}$ after haloperidol, quetiapine or clozapine injections (Fig. 1a,b, respectively). These drugs also induced significant impairments in the ability to clear a glucose load. The effect was greatest for the SGA drugs, with haloperidol having only a minor effect.

The effects of repeated SGA dosing on glucose tolerance To determine whether repeated daily dosing altered the acute effects of these drugs on glucose metabolism, rats were injected daily for 7 or 28 days. After such dosing, acute administration of both quetiapine and clozapine was still able to significantly impair glucose disposal (ESM Fig. 2a, c, respectively) and to increase plasma insulin levels during a GTT (ESM Fig. 2b,d, respectively). However, after repeated dosing of haloperidol this drug was no longer able to have a significant effect on glucose metabolism.

To determine the persistence of the effect observed on glucose sensitivity, we performed a GTT $48 \mathrm{~h}$ and 7 days after the last drug injection, in rats previously exposed to antipsychotic medication for 28 consecutive days. A small but significant defect in glucose tolerance remained $48 \mathrm{~h}$ after the final injection with quetiapine or clozapine exposure (ESM Fig. 2e). However, this difference was normalised by 7 days following drug removal (ESM Fig. 2f). Thus, the impairment in glucose tolerance caused by SGAs is not permanent and can be reversed by drug removal.

SGAs do not acutely induce insulin resistance The impaired glucose tolerance associated with increased insulin secretion observed following SGA treatment in humans is classically interpreted as a consequence of an obesityinduced insulin-resistant state. In the present study, no significant weight gain in the animals was observed in either the 7 day or 28 day treatment protocols. Further, the effects can be rapidly reversed by drug removal, suggesting the effects we see are largely caused by an acute affect on glucose use.

To test whether SGAs caused insulin resistance we first studied the effect of the drugs on glucose uptake in fat and 
muscle, as these tissues are two major sites of insulinstimulated glucose disposal in the periphery. As expected, wortmannin, a phosphatidylinositol 3-kinase inhibitor, significantly reduced glucose uptake after insulin stimulation in 3T3L1 adipocytes (Fig. 2a). However, pretreatment of 3T3L1 adipocytes with all three drugs at therapeutically relevant concentrations $(1 \mu \mathrm{mol} / \mathrm{l})$ did not modify insulinstimulated glucose uptake. At $10 \mu \mathrm{mol} / \mathrm{l}$, levels of drugs at least tenfold higher than that achieved therapeutically, a marginal decrease of glucose uptake was observed. We next studied the effect of these drugs on glucose uptake in soleus muscle. As expected wortmannin significantly reduced glucose uptake (Fig. 2b). However, a pre-incubation of
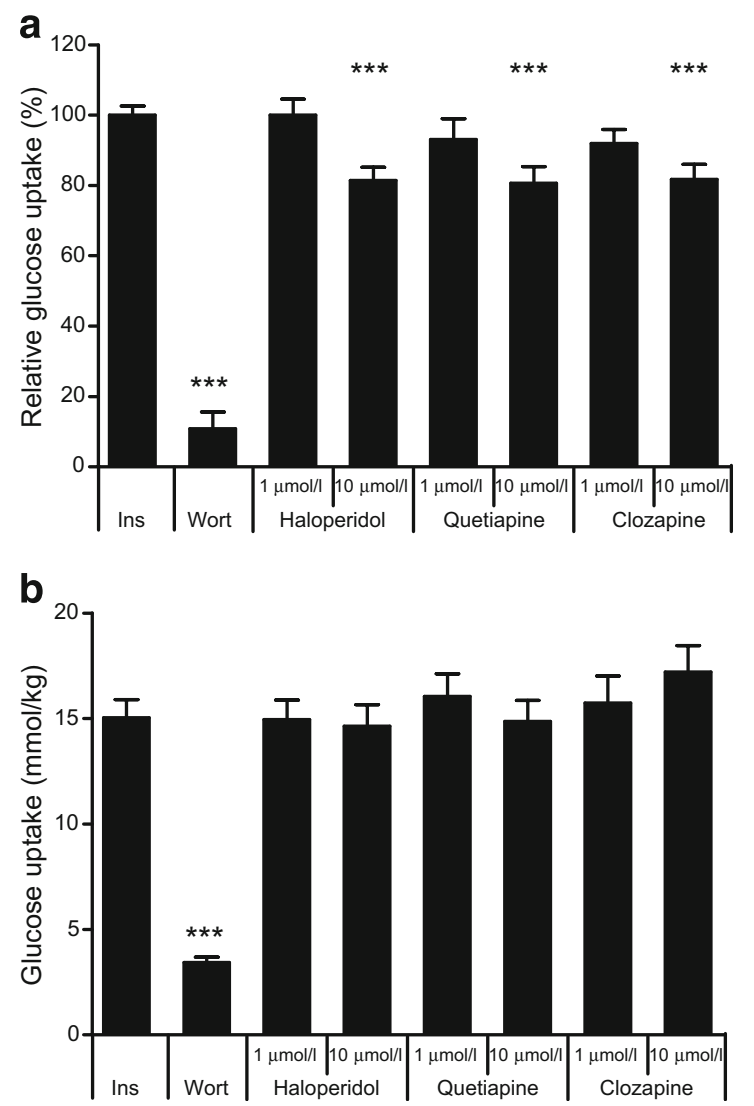

Fig. 2 The effect of antipsychotic medication on glucose uptake in 3T3L1 adipocytes and soleus muscle. a 3T3L1 adipocytes were starved overnight and incubated for $30 \mathrm{~min}$ with wortmannin (Wort; $100 \mathrm{nmol} / \mathrm{l}$ ) or the antipsychotic drugs (at the concentrations indicated) prior to insulin (Ins) stimulation (100 nmol/l, $10 \mathrm{~min}$ ). Glucose uptake was performed by addition of 2-deoxy-D- $\left[{ }^{3} \mathrm{H}\right]$ glucose for $20 \mathrm{~min}$. The reaction was stopped by aspiration and followed by three washes with ice-cold PBS. Cells were lysed with $0.5 \mathrm{~mol} / 1$ $\mathrm{NaOH}$ followed by $0.5 \mathrm{~mol} / 1 \mathrm{HCl}$ and the incorporated radioactivity measured using a liquid scintillation counter. Results are means \pm SEM for three independent experiments each performed in four replicate experiments. b Glucose uptake in soleus muscle isolated from the rat, $n=6$ per group. $* * * p<0.001$ vs the insulin only group by one-way ANOVA and Dunnett's multiple-comparison test the drugs did not cause any defect in glucose uptake even at $10 \mu \mathrm{mol} / 1$ concentration. This demonstrated that relevant concentrations of these drugs are not directly causing insulin resistance in two major insulin target tissues.

We next examined the effect on insulin signalling in liver and fat using the phosphorylation of $\mathrm{Akt} / \mathrm{PKB}$ on serine 473 as a marker of insulin action. Rat tissue was collected $2 \mathrm{~h}$ after the last drug injection, at which time both glucose and insulin levels were raised with clozapine (Fig. 3a,c, respectively). In the liver, we found a significant increase in the phosphorylation of serine 473 on Akt/PKB with rats injected with clozapine (Fig. 3b). Likewise, we found a tenfold increase in serine 473 phosphorylation on Akt/PKB in the retroperitoneal fat of rats injected with clozapine (Fig. 3d). No change in total levels of Akt/PKB was found. Thus, the activity of a downstream target of insulin, $\mathrm{Akt} / \mathrm{PKB}$, is not inhibited by clozapine, again demonstrating this drug is not causing direct impairment of insulin signalling in the rat.

To further investigate the effect of SGAs on insulin sensitivity, we performed ITT in drug-naive animals. We found no defect in insulin sensitivity following $1 \mathrm{~h}$ drug exposure for all drugs tested (Fig. 4). This provides further evidence that the increases in glucose and insulin levels during the GTT following acute administration of either quetiapine or clozapine are not caused by the development of underlying insulin resistance.

Investigating the effects of SGAs on HGO The observation that blood glucose levels are increasing in the absence of insulin resistance suggests that the drugs could be stimulating HGO. Pyruvate and glycerol are two major substrates for gluconeogenesis, so to determine if there was increased glucose production we performed both PTT and GlycTT after acute drug administration. We found a significant increase in AUC in both PTT and GlycTT in rats exposed to haloperidol, quetiapine or clozapine (Fig. 5a,e, respectively). Because clozapine increases basal blood glucose level we normalised the blood glucose levels to rats that received the drugs only (Fig. 5b,f). This allowed us to estimate the amount of glucose being produced by either pyruvate or glycerol (Fig. 5c,g). As for the effect on GTT, this effect was transient as, by $48 \mathrm{~h}$ after the acute drug administration, no change in either PTT or GlycTT was observed (Fig. 5d,h). In conclusion, these data provide new evidence that SGAs are acutely promoting glucose production in the liver.

The increased HGO is surprising as we have shown that these drugs are acutely increasing glucose and insulin levels and both of these normally act to suppress HGO. As the stimulation of $\mathrm{Akt} / \mathrm{PKB}$ phosphorylation in the liver remains unchanged, insulin resistance is an unlikely explanation. Therefore the most likely explanation was that the SGAs were inducing glucagon secretion despite the 
Fig. 3 The effect of antipsychotic medication on Akt/PKB activity. Blood glucose (a) and plasma insulin (c) levels following $2 \mathrm{~h}$ drug injection. Western blot analysis and quantification showing the effect of antipsychotic drugs on the activity (phosphorylated serine 473 [p-S473] on Akt/PKB) and total levels of $\mathrm{Akt} / \mathrm{PKB}$ in the liver (b) and retroperitoneal fat (d). Data were calculated as a ratio of serine 473 (p-S473) to total Akt/PKB for each sample and expressed as fold change relative to the vehicle control group ( $n=6$ per group). Data are means \pm SEM. $* * * p<0.001$ vs the vehicle control group determined by one-way ANOVA and Fisher's post hoc test
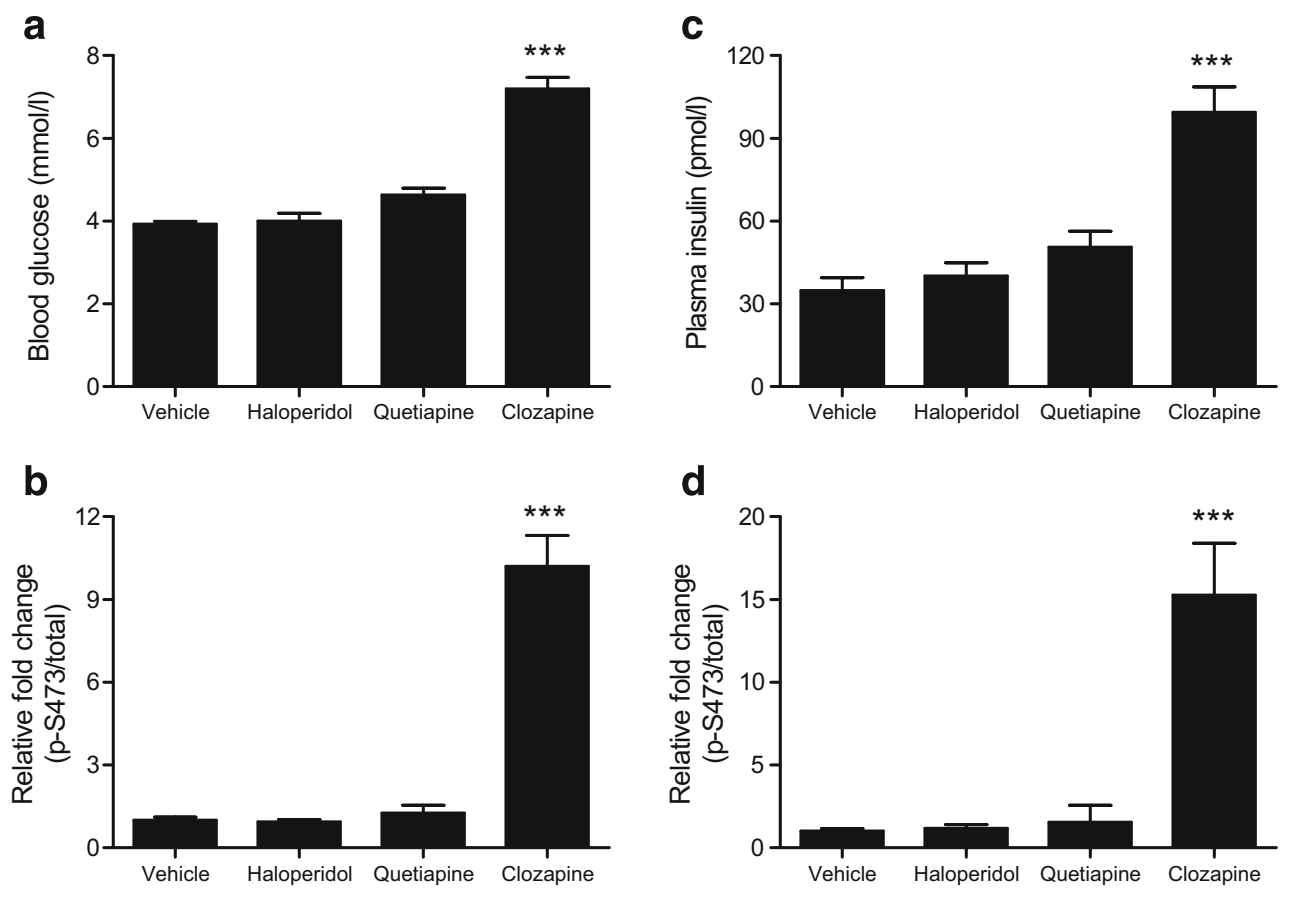

d
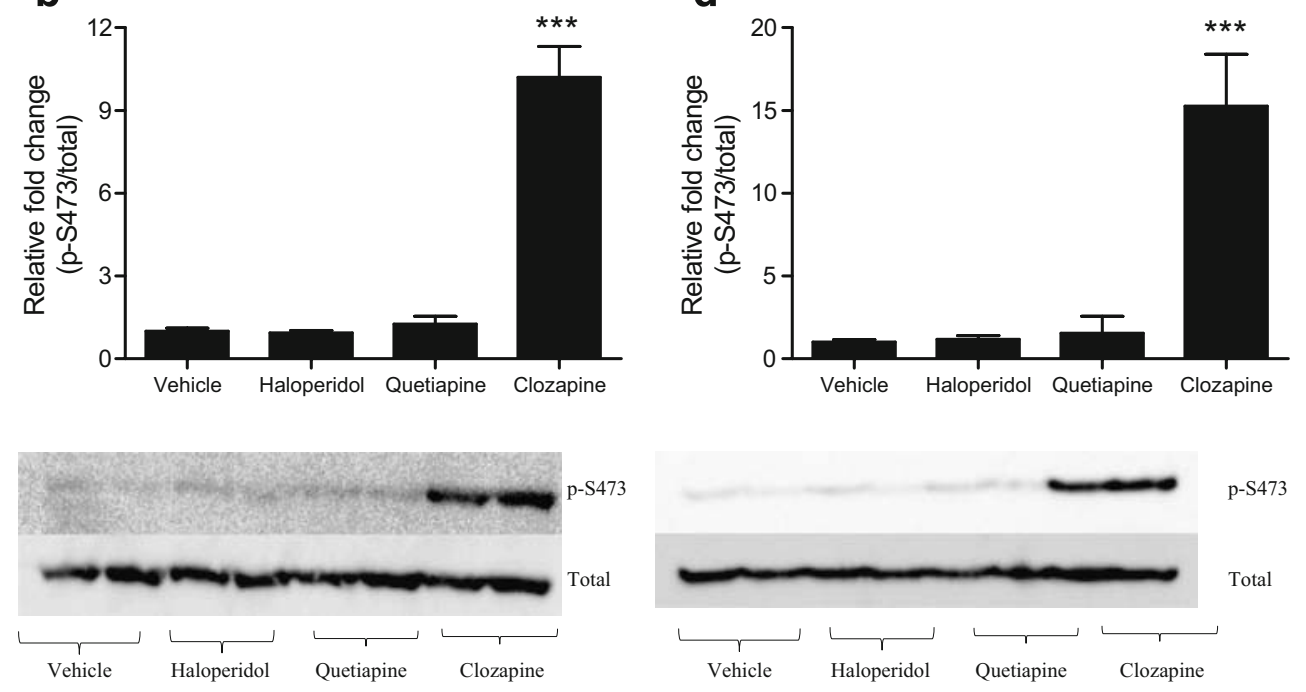

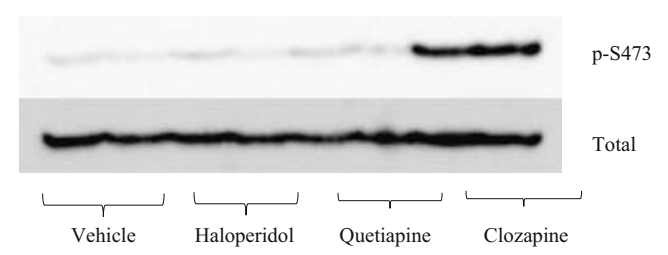

high glucose and insulin levels. When blood glucose rises, glucagon secretion is normally shut down and its actions are counteracted by insulin. However, following clozapine injection, glucagon levels actually increase further (Fig. 6a) despite increases in both blood glucose and plasma insulin levels (Fig. 6b,c, respectively), suggesting clozapine is directly promoting glucagon secretion. In control experiments we confirmed that administration of glucose to control rats induced increases in blood glucose and insulin while almost totally suppressing glucagon secretion (data not shown). To more fully understand the role of glucagon in the clozapine effect we performed experiments using octreotide, a somatostatin analogue that abolishes glucagon and insulin secretion (Fig. 6). These two effects would be expected to have opposing effects on glucose metabolism with falling insulin expected to increase blood glucose levels and falling glucagon expected to reduce blood glucose levels. The fact that octreotide treatment significantly attenuates the effects of clozapine on increasing blood glucose levels supports the conclusion that the clozapineinduced increase in glucagon is the major contributor to the elevated blood glucose levels in SGA-treated animals.

We also found that octreotide attenuates the elevation in glucose concentration after a pyruvate or glycerol load
(Fig. 7a,b, respectively). We studied the effect of clozapine at $90 \mathrm{~min}$ after pyruvate and $60 \mathrm{~min}$ after glycerol exposure on blood glucose levels with or without octreotide. As expected, vehicle rats injected with octreotide/pyruvate or

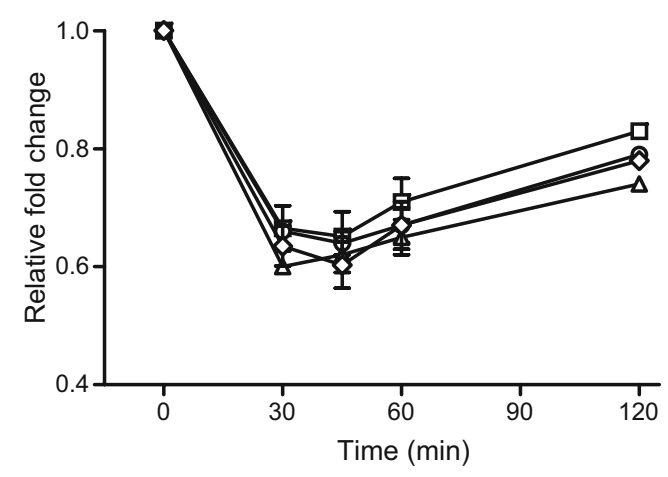

Fig. 4 The effect of antipsychotic medication on insulin sensitivity during an ITT. Rats were weight-matched into groups and fasted for $2 \mathrm{~h}$ prior to drug or vehicle injection. Following $1 \mathrm{~h}$ drug exposure, insulin $(0.75 \mathrm{U} / \mathrm{kg})$ was injected s.c. and blood glucose concentrations monitored at the indicated time points. Data were normalised to the blood glucose measurement at time 0 for each group to calculate a relative fold-change as shown in the results. Squares, vehicle; circles, haloperidol; triangles, quetiapine; diamonds, clozapine. $n=6$ per group. Data are means \pm SEM 

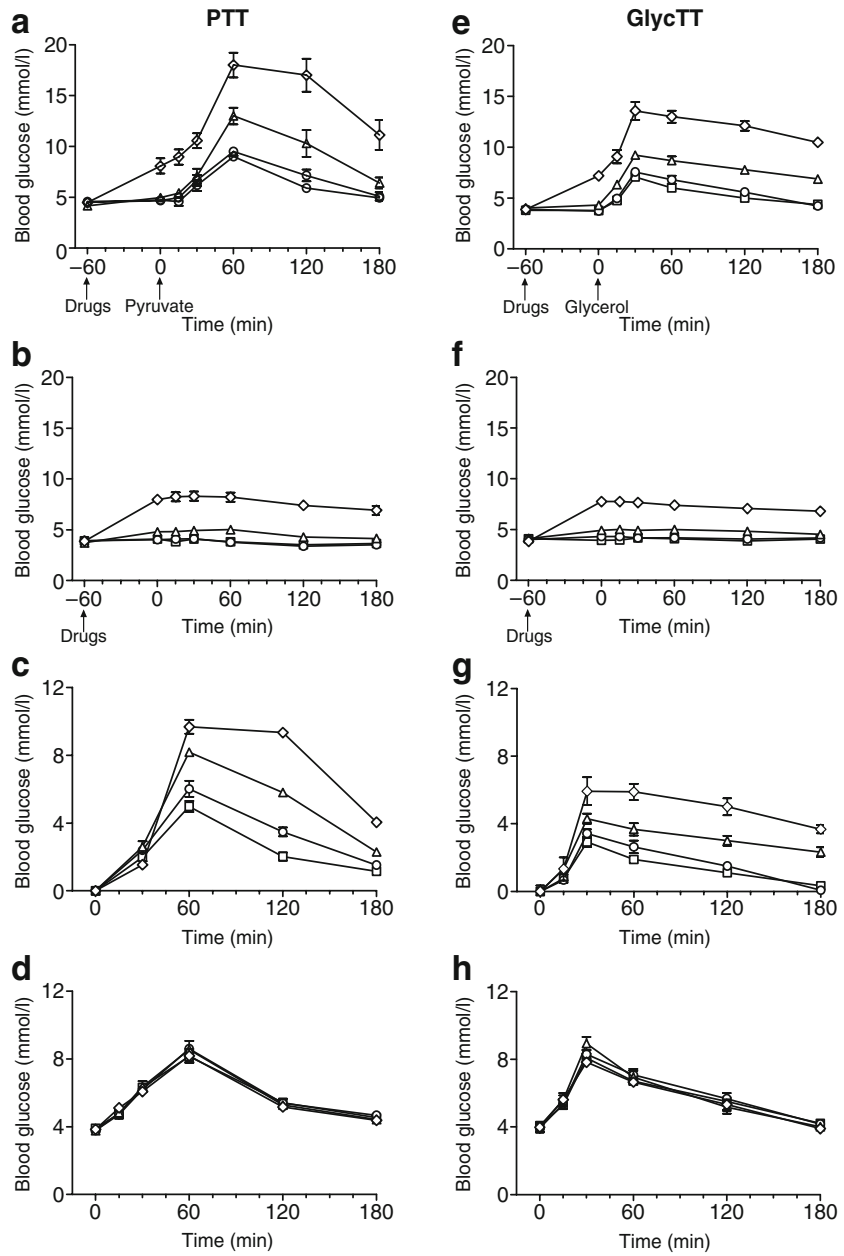

Fig. 5 The acute effect of antipsychotic drugs on blood glucose levels in response to pyruvate $(\mathbf{a}-\mathbf{d})$ or glycerol $(\mathbf{e}-\mathbf{h})$. a, e Blood glucose $1 \mathrm{~h}$ after drug injection. $\mathbf{b}$, f Basal blood glucose levels after drug treatment only. c, $\mathbf{g}$ Blood glucose levels of rats injected with drugs then injected with pyruvate or glycerol (a, e) minus rats treated with drugs only $(\mathbf{b}, \mathbf{f})$. d, h Blood glucose levels $48 \mathrm{~h}$ after acute drug injection. Vehicle (squares), haloperidol (circles), quetiapine (triangles) and clozapine (diamonds). ( $n=9$ per group). Data are means \pm SEM. a, c, e, $\mathbf{g} p<0.001$ for quetiapine and clozapine, $\mathbf{b}, \mathbf{f} p<0.001$ for clozapine vs the vehicle control group by repeated-measures ANOVA

octreotide/glycerol had a 1.6-fold and a 1.4-fold increase in blood glucose levels, respectively (Fig. 7). Interestingly, the presence of octreotide decreased blood glucose levels in clozapine/pyruvate- or clozapine/glycerol-injected rats. This is consistent with the effects of clozapine being mediated via glucagon secretion, as glucagon would normally promote the conversion of pyruvate and glycerol to glucose.

\section{Discussion}

SGAs are a widely used class of drug, but one side effect of these drugs is the development of a type 2 diabetes-like phenotype characterised by high circulating blood glucose and insulin, especially with clozapine and olanzapine [2, 6, 9, 10, 22]. In humans, as the use of SGAs is associated with weight gain, the assumption has been made that these drugs are causing the development of severe insulin resistance in the major insulin-sensitive tissues, inducing compensatory increases in insulin secretion [3]. Indeed, some previous studies have claimed SGAs induce insulin resistance in cell culture models, albeit using the drugs at supra-therapeutic concentrations $[6-8,11]$.

In the current animal studies, we have deliberately used dosing regimes that achieve therapeutically relevant
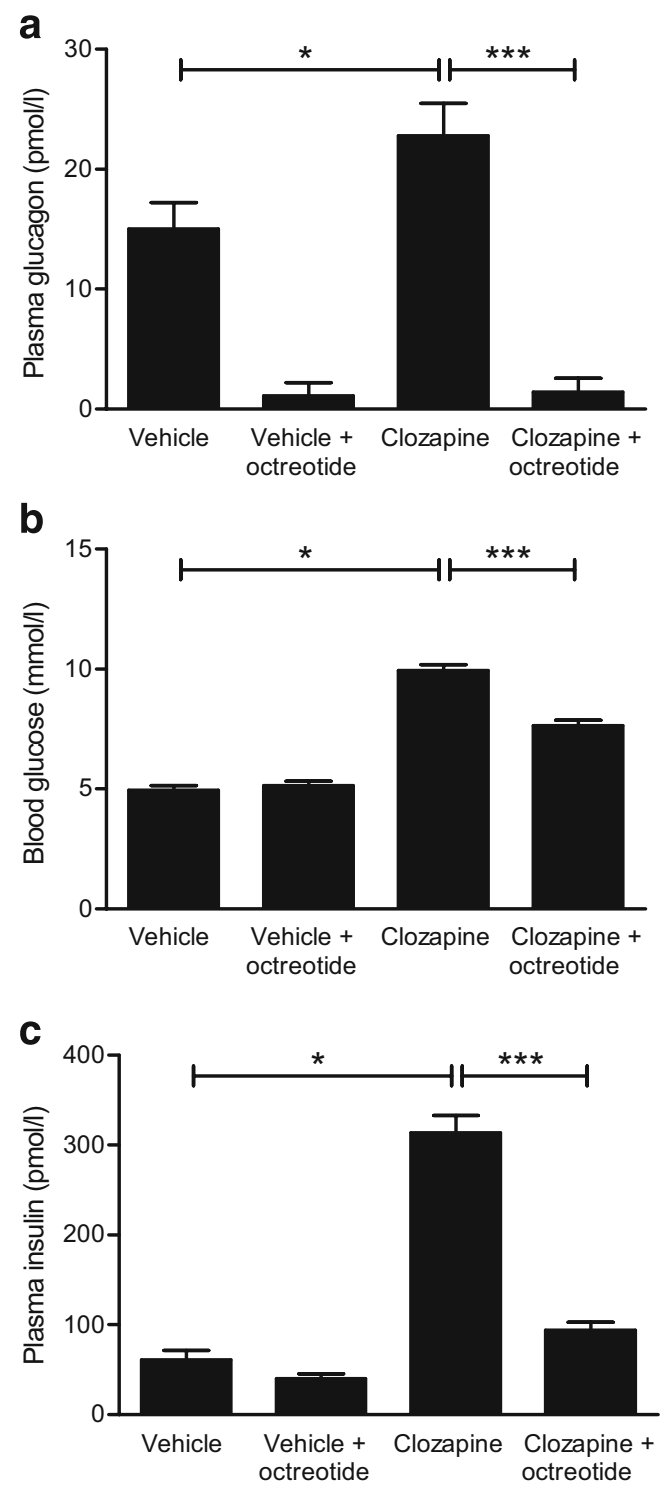

Fig. 6 The effect of clozapine on glucagon secretion. a Plasma glucagon levels $1 \mathrm{~h}$ after clozapine and clozapine plus octreotide injections. Blood glucose levels (b) and plasma insulin levels (c) during clozapine and octreotide injections. ( $n=9$ per group). Data are means \pm SEM. ${ }^{*} p<0.05, * * * p<0.001$ vs the indicated comparison group by one-way ANOVA and Fisher's post hoc test 

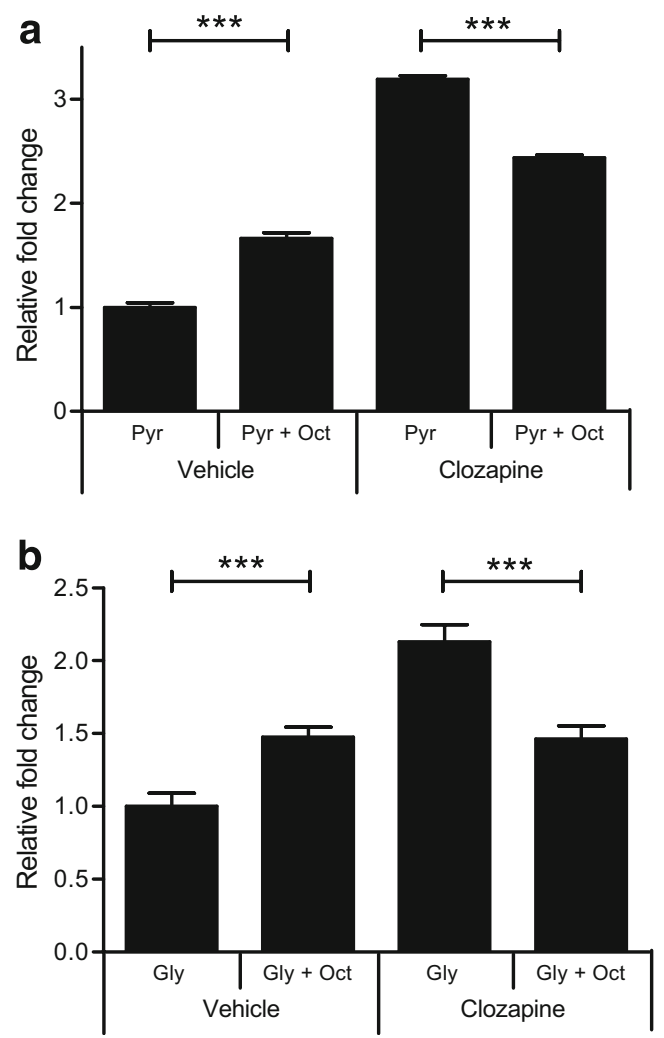

Fig. 7 The effect of octreotide on blood glucose levels following pyruvate (a) or glycerol (b) exposure. Relative fold change of blood glucose levels in rats injected with vehicle or clozapine with or without pyruvate or glycerol plus octreotide (Oct) after $90 \mathrm{~min}$ (pyruvate) or $60 \mathrm{~min}$ (glycerol) exposure (relative to pyruvate or glycerol vehicle, respectively). $n=9$ per group. Data are means \pm SEM. $* * p<0.01, * * * p<0.001$ vs the indicated comparison group by repeated-measures ANOVA Fisher's post hoc test

plasma levels of drugs in animals and under these conditions we do find that all three antipsychotic medications examined cause impairments of glucose metabolism. The effects were greatest for the two SGAs tested (clozapine and quetiapine). Surprisingly these effects were independent of any effects on increased body weight or adiposity and could be observed rapidly after drug administration.

While we find evidence that a small degree of insulin resistance may exist after long-term administration of SGA, we provide several lines of evidence indicating that the acute effects of these drugs on glucose metabolism are not caused by an induction of insulin resistance. First, we did not identify any impairment of insulin action in both adipocytes and isolated soleus muscle using therapeutically relevant drug concentrations, and only minor effects at supra-therapeutic levels in adipocytes. Further, the impaired glucose tolerance after chronic drug exposure did not persist after drug removal, indicating that the drugs were not inducing long-term changes in the metabolism of the animals. The finding that there is still strong activation of
Akt/PKB in insulin responsive tissue (fat and liver) when drug is administered also provides evidence that the animals were not in fact insulin-resistant. This was further confirmed by the fact that none of the drugs altered glucose clearance rates in ITT.

If these drugs are not acutely inducing insulin resistance but inducing an increase in glucose and insulin levels after acute drug exposure, the most obvious explanation is that they are stimulating HGO and as a consequence stimulating insulin release. The production of glucose from the liver could be achieved by either increasing gluconeogenesis and/or stimulating glycogenolysis. Our finding that, compared with control, the SGAs increase glucose levels following either pyruvate or glycerol administration, after adjusting for any increased basal blood glucose levels because of the drugs only, provides evidence that the drugs are at least acting to promote gluconeogenesis. In the case of clozapine our data suggest these effects are caused by an increase in glucagon secretion, because following inhibition of both insulin and glucagon secretion during $90 \mathrm{~min}$ of pyruvate or $60 \mathrm{~min}$ of glycerol we found that animals injected with clozapine/pyruvate/octreotide or clozapine/ glycerol/octreotide decreased HGO compared with clozapine/pyruvate- or clozapine/glycerol-exposed animals. Insulin levels are greatly reduced in these animals, which would normally enable increases in HGO, so the fact that there is a reduction can most likely be attributed to a decrease in glucagon secretion caused by octreotide. To further support this we also find that clozapine acts to increase glucagon levels despite concurrent increases in blood glucose and insulin levels, a situation which would normally reduce glucagon levels.

In summary our study identifies a previously undescribed acute effect of SGAs on glucose metabolism in which a diabetes-like phenotype is caused via a stimulation of glucagon secretion, which subsequently impacts on $\mathrm{HGO}$, increasing blood glucose and thus insulin levels. It is already known that long-term treatment of humans with SGAs leads to weight gain, which will induce insulin resistance in peripheral tissues. If the acute mechanism described here is also operating in already insulin-resistant SGA-treated patients then it will almost certainly make a significant contribution to the type 2 diabetes phenotype observed in this group. To the best of our knowledge there are no studies addressing the acute effects of SGA administration on glucose metabolism and its relationship to glucagon levels in humans. However, there is one study reporting the effects of chronic SGA treatment on glucose, insulin and glucagon levels in humans during a GTT [23]. This study reported that despite large increases in glucose and insulin levels, there was no suppression of glucagon levels in individuals treated with SGAs. This is consistent with the effects we observe in rats, but further studies will 
be required to determine whether the SGAs directly affect glucagon secretion and HGO in humans.

Acknowledgements This work was supported by grants from Lottery Health and the Oakley Mental Health Research Foundation, New Zealand. Additional funding was provided by the Health Research Council of New Zealand and by the Novo Nordisk Foundation and the European Commission via COST BM602. The authors thank E. Thorstensen for his technical support for the quantification of the drugs using HPLC and Douglas Pharmaceutical (clozapine) and AstraZeneca (quetiapine) for supplying us with antipsychotic compounds. We also thank A. Ingvaldsen and J. T. Stuenaes for technical assistance.

Duality of interest The authors declare that there is no duality of interest associated with this manuscript.

\section{References}

1. Albaugh VL, Henry CR, Bello NT et al (2006) Hormonal and metabolic effects of olanzapine and clozapine related to body weight in rodents. Obesity 14:36-53

2. Newcomer JW (2007) Antipsychotic medications: metabolic and cardiovascular risk. J Clin Psychiatry 68:8-13

3. Allison DB, Montore JL, Heo M et al (1999) Antipsychoticinduced weight gain: a comprehensive research synthesis. Am J Psychiatry 156:1686-1696

4. Henderson DC, Cagliero E, Gray C et al (2000) Clozapine, diabetes mellitus, weight gain, and lipid abnormalities: a five-year naturalistic study. Am J Psychiatry 157:975-981

5. Dwyer DS, Donohoe D (2003) Induction of hyperglycemia in mice with atypical antipsychotic drugs that inhibit glucose uptake. Pharmacol Biochem Behav 75:255-260

6. Vestri HS, Maianu L, Moellering DR, Garvey WT (2007) Atypical antipsychotic drugs directly impair insulin action in adipocytes: effects of glucose transport, lipogenesis and antilipolysis. Neuropsychopharmacology 32:765-772

7. Ardizzone TD, Bradley RJ, Freeman AM, Dwyer DS (2001) Inhibition of glucose transport in $\mathrm{PC} 12$ cells by the atypical antipsychotic drugs risperidone and clozapine, and structural analogs of clozapine. Brain Res 923:82-90

8. Dwyer DS, Pinkofsky HB, Liu Y, Bradley RJ (1999) Antipsychotic drugs affect glucose uptake and the expression of glucose transporters in PC12 cells. Prog Neuropsychopharmacol Biol Psychiatry 23:69-80

9. Murashita M, Kusumi I, Hosoda H, Kangawa K, Koyama T (2007) Acute administration of clozapine concurrently increases blood glucose and circulating plasma ghrelin levels in rats. Psychoneuroendocrinology 32:777-784
10. Tulipano G, Rizzetti C, Bianchi I, Fanzani A, Spano P, Cocchi D (2007) Clozapine-induced alteration of glucose homeostasis in the rat: the contribution of hypothalamic-pituitary-adrenal axis activation. Neuroendocrinology 85:61-70

11. Engl J, Laimer M, Niederwanger A et al (2005) Olanzapine impairs glycogen synthesis and insulin signaling in L6 skeletal muscle cells. Mol Psychiatry 10:1089-1096

12. Kapur S, VanderSpek SC, Brownlee BA, Nobrega JN (2003) Antipsychotic dosing in preclinical models is often unrepresentative of the clinical condition: a suggested solution based on in vivo occupancy. J Pharmacol Exp Ther 305:625-631

13. Titier K, Bouchet S, Pehourcq F, Moore N, Molimard M (2003) High-performance liquid chromatographic method with diode array detection to identify and quantify atypical antipsychotics and haloperidol in plasma after overdose. J Chromatogr B Analyt Technol Biomed Life Sci 788:179-185

14. Sachse J, Koller J, Hartter S, Hiemke C (2006) Automated analysis of quetiapine and other antipsychotic drugs in human blood by high performance liquid chromatography. J Chromatogr B Analyt Technol Biomed Life Sci 830:342-348

15. Chaussade C, Rewcastle GW, Kendall JD et al (2007) Evidence for functional redundancy of class IA PI3K isoforms in insulin signalling. Biochem J 404:449-458

16. Navé BT, Haigh RJ, Hayward AC, Siddle K, Shepherd PR (1996) Compartment-specific regulation of phosphoinositide 3-kinase by platelet-derived growth factor and insulin in 3T3-L1 adipocytes. Biochem J 318:55-60

17. Whitehead JP, Soos MA, Aslesen R, O'Rahilly S, Jensen J (2000) Contraction inhibits insulin-stimulated insulin receptor substrate1/2-associated phosphoinositide 3-kinase activity, but not protein kinase B activation or glucose uptake, in rat muscle. Biochem $\mathrm{J}$ 349:775-781

18. Jensen J, Jebens E, Brennesvik EO et al (2006) Muscle glycogen inharmoniously regulates glycogen synthase activity, glucose uptake, and proximal insulin signaling. Am J Physiol 290:154162

19. Citrome L, Volavka J (2002) Optimal dosing of atypical antipsychotics in adults: a review of the current evidence. Harv Rev Psychiatry 10:280-291

20. Kapur S, Zipursky RB, Remington G (1999) Clinical and theoretical implications of 5-HT2 and D2 receptor occupancy of clozapine, risperidone and olanzapine in schizophrenia. Am J Psychiatry 156:286-293

21. Tauscher Y, Kapur S (2001) Choosing the right dose of antipsychotic in schizophrenia: lessons from neuroimaging studies. CNS Drugs 15:671-678

22. Zhao Z, Ksiezak-Reding H, Riggio S, Haroutunian V, Pasinetti GM (2006) Insulin receptor deficits in schizophrenia and in cellular and animal models of insulin receptor dysfunction. Schizophr Res 84:1-14

23. Newcomer JW, Haupt DW, Fucetola R et al (2002) Abnormalities in glucose regulation during antipsychotic treatment of schizophrenia. Arch Gen Psychiatry 59:337-345 